\title{
FROM HILBERT PROOFS TO CONSECUTIONS AND BACK
}

\author{
TORE FJETLAND ØGAARD
}

Abstract: Restall presented in An Introduction to Substructural Logics a "consecution" calculus. This is a natural deduction type sequent calculus where the structural rules play an important role. This paper looks at different ways of extending Restall's calculus. It is shown that Restall's weak soundness and completeness result with regards to a Hilbert calculus can be extended to a strong one so as to encompass what Restall calls proofs from assumptions. It is also shown how to extend the calculus so as to validate the metainferential rule of reasoning by cases, as well as certain theory-dependent rules.

Keywords: · consecution - external consequence $\cdot$ Hilbert consequence $\cdot$ relevant logic $\cdot$ substructural proof theory

\section{Contents}

1. Introduction 52

2. Consecution and Hilbert proofs for $\mathbf{B} \quad 52$

3. Translations between calculi 56

4. Generalizing to other logics 61

4.1. A stronger falsum 61

4.2. A stronger negation $\quad 62$

4.3. Adding structural rules $\quad 63$

4.4. More connectives 63

5. Generalizing further: Adding meta-inferences - the case of reasoning by cases in substructural proof theory $\quad 64$

5.1. Other meta-rules 69

6. Generalizing further still: adding theory-dependent rules 69

$\begin{array}{ll}7 . & \text { Summary } \\ & 70\end{array}$

$\begin{array}{ll}\text { References } & 70\end{array}$

Date: April 30, 2021.

I would very much like to thank Andreas Fjellstad for engaging discussion in an early stage of this project, as well as Shawn Standefer and the anonymous referee for helpful comments and suggestions on a later draft.

Australasian Journal of Logic (18:2) 2021, Article no. 2 


\section{INTRODUCTION}

Restall presented in his book An Introduction to Substructural Logics (Restall, 2000) a "consecution" calculus for logics in the vicinity of relevant logics. Restall's calculus is a type of natural deduction calculus but where the proof objects are not formulas but rather consecutions, more commonly known as sequents. ${ }^{1}$ Restall proved that a weak soundness and completeness result holds for all logics dealt with by him to the effect that a consecution is provable in some logic if and only if its translation into a formula is a logical theorem of the Hilbert calculus for the logic in question. Soundness and completeness result are called weak if they only apply to derivations from the logical axioms, but are called strong if they also apply to derivations from non-logical premises. In this paper it is proven that Restall's result can be strengthened to a strong soundness and completeness result. This is proven in some detail for the weak relevant logic $\mathbf{B}$. The proof holds for many logics, however, but to keep the paper from growing out of proportions it will only be gestured at how the result in fact generalizes. Section 1-3 contains the result for $\mathbf{B}$ and Section 4 the gesturing towards the plethora of other logics dealt with in Restall's book.

Relevant logics are often strengthened by certain metainferential rules, and in the propositional case, specifically the meta-rule of reasoning by cases. Section 5 shows how to extend Restall's consecution calculus so as to yield a strong soundness and completeness result also with regards to $\mathbf{B}^{d}-\mathbf{B}$ with reasoning by cases added. It will be evident from the proof that the result generalizes to stronger logics than $\mathbf{B}^{d}$.

Non-classical logics are, when logical consequence is defined in the usual Hilbertian way, often fitted with what are in this paper called theory-dependent rules. For instance, it is sometimes argued that the induction schema for Peano arithmetic is too strong in the context of certain logics if stated as an axiom schema. To retain "enough" induction, one then rather includes a primitive induction rule. Section 6 shows what the consecution-equivalent of adding such theory-dependent rules is. From the previous result it will then follow that strong soundness and completeness holds also for this kind of extension. Section 7 briefly summarises.

This is first and foremost but a technical paper on the relationship between two different proof calculi. For a more general overview of both technical as well as philosophical issues connected to relevant and substructural logics, see Bimbó (2007), Dunn and Restall (2002), Mares and Meyer (2001), and Restall (2006).

\section{CONSECUtion ANd Hilbert PROOFs For B}

I will refer to $\vdash_{\mathbf{L}}^{h}$ as Hilbert consequence for the logic $\mathbf{L}$. The consequence relation is defined as follows:

\footnotetext{
${ }^{1}$ Variants of this type of calculus can be found in Read (1988), Slaney (1990), and Restall (1994). As pointed out by the referee, Restall's consecution calculus actually comes in two different variants: the natural deduction one as well as a Gentzen variant (see Restall (2000, ch. 6)). Whether or not similar results as those presented in this paper can also be given in the case of the Gentzen variant will have to be tackled in a different paper, however.
} 
Definition 1. [The Hilbert consequence relation of a logic] A HILBERT PROOF of a formula $A$ from a set of formulas $\Gamma$ in the logic $\boldsymbol{L}$ is defined to be a finite list $A_{1}, \ldots, A_{n}$ such that $A_{n}=A$ and every $A_{i \leq n}$ is either a member of $\Gamma$, a logical axiom of $\boldsymbol{L}$, or there is a set $\Delta \subseteq\left\{A_{j} \mid j<i\right\}$ such that $\Delta \Vdash A_{i}$ is an instance of a rule of $\boldsymbol{L}$. The existential claim that there is such a proof is written $\Gamma \vdash_{\mathbf{L}}^{h} A$ and expressed as "there exists a Hilbert-derivation of A from $\Gamma$ in the logic $\mathbf{L}$."

Definition 2 (Parenthesis conventions). The basic set of connectives considered in this paper will be $\{\perp, \mathbf{t}, \sim, \vee, \wedge, \circ, \rightarrow\}$, where the first two are nullary (truthconstants), the third unary, and the remaining binary connectives. The disjunction $\vee$, and the conjunctions $\wedge$ and $\circ$ are to bind tighter than $\rightarrow$, and so I'll usually drop parenthesis enclosing conjunctions and disjunctions.

Definition 3. The following list of axioms and rules defines the logic $\boldsymbol{B}$ :

$$
\begin{array}{ll}
(A 1) & A \rightarrow A \\
(A 2) & A \rightarrow A \vee B \text { and } B \rightarrow A \vee B \\
(A 3) & A \wedge B \rightarrow A \text { and } A \wedge B \rightarrow B \\
(A 4) & A \wedge(B \vee C) \rightarrow(A \wedge B) \vee(A \wedge C) \\
(A 5) & (A \rightarrow B) \wedge(A \rightarrow C) \rightarrow(A \rightarrow B \wedge C) \\
(A 6) & (A \rightarrow C) \wedge(B \rightarrow C) \rightarrow(A \vee B \rightarrow C) \\
(A 7) & \sim \sim A \rightarrow A \\
(A \perp) & \perp \rightarrow A \\
(A \circ) & A \rightarrow(B \rightarrow A \circ B) \\
(A \mathbf{t}) & \mathbf{t} \\
\hline(R 1) & \{A, A \rightarrow B\} \Vdash B \\
(R 2) & \{A, B\} \Vdash A \wedge B \\
(R 3) & \{A \rightarrow B\} \Vdash(C \rightarrow A) \rightarrow(C \rightarrow B) \\
(R 4) & \{A \rightarrow B\} \Vdash(B \rightarrow C) \rightarrow(A \rightarrow C) \\
(R 5) & \{A \rightarrow \sim B\} \Vdash B \rightarrow \sim A \\
(R \circ) & \{A \rightarrow(B \rightarrow C)\} \Vdash A \circ B \rightarrow C \\
(R \mathbf{t}) & \{A\} \Vdash \mathbf{t} \rightarrow A
\end{array}
$$

The axiomatization of $\mathbf{B}$ is slightly different from the setup in Restall (2000, ch. 4). First of all, the pre- and suffixing rules (R3) \& (R4) are rather stated as the affixing rule $\{A \rightarrow B, C \rightarrow D\} \Vdash(B \rightarrow C) \rightarrow(A \rightarrow D)$. Furthermore, Restall's rules for $\mathbf{t}$ - the truth-constant known as the Ackermann constant - is (Rt) and instead of (At), the rule $\{\mathbf{t} \rightarrow A\} \Vdash A$. It is an easy task to verify that the different choices are in fact equivalent - a task I leave with the reader. Restall's rules for $\circ$ - the fusion connective- is the rule (Ro), and instead of (Ao), rather the rule $\{A \circ B \rightarrow C\} \Vdash A \rightarrow(B \rightarrow C)$. That $(\mathrm{A} \circ)$ is derivable using Restall's rule is evident. That the rule is derivable using $(\mathrm{A} \circ)$ is seen from the following which we will refer back to later:

Australasian Journal of Logic (18:2) 2021, Article no. 2 


\section{Lemma 1.}
(1) $A \circ B \rightarrow C$
assumption
(2) $(B \rightarrow A \circ B) \rightarrow(B \rightarrow C)$
$1, R 3$
(3) $(A \rightarrow(B \rightarrow A \circ B)) \rightarrow(A \rightarrow(B \rightarrow C)) 2, R 3$
(4) $A \rightarrow(B \rightarrow A \circ B)$
$A \circ$
(5) $A \rightarrow(B \rightarrow C)$
$3,4 R 1$

I have chosen to take the Church constant $\perp$ as primitive. It's positive sibling $T$ is axiomatized by the single axiom $A \rightarrow \mathrm{T}$, but in the presence of the contraposition rule (R5), $T$ can be regarded as defined with $T=d f \sim \perp .^{2}$ The axiom (A $\perp$ ) is the most common way of axiomatizing $\perp$. Note that Restall (2000)'s consecution-rule for $\perp$, however, uses a stronger condition which in the case of $\mathbf{B}$ corresponds to adding in addition the axiom $C \rightarrow(\perp \rightarrow \perp)$. I'll show in Section 4 that the added axiom for $\perp$ is precisely what is needed to translate Restall's rule for $\perp$.

Now for Restall's consecution calculus. I follow Restall in using 'consecution' instead of the more common 'sequent.' I use ' $\triangleright$ ' where Restall uses ' $r$ ' so as to distinguish clearly between consecutions and a consequence relation: I will therefore use ' $\triangle$ ' for consecutions and variants of ' $\vdash$ ' for consequence relations. ' $\Vdash$ ' and ' $\Leftarrow$ ' will be used for rules.

\section{Definition 4. (Structure)}

- 0 is a structure (but not a formula)

- If A is a formula, then A is a structure

- If $X$ and $Y$ are structures, then so is $(X, Y)$

- If $X$ and $Y$ are structures, then so is $(X ; Y)$

- If $X$ is a structure, and $A$ is a formula, then $X \triangleright A$ is a consecution.

Substructure is defined in the obvious way.

- $X(Y)$ indicates that $Y$ is a substructure of $X$.

- $X(Y / Z)$ is the structure obtained by replacing every substructure $Y$ in $X$ with $Z .^{3}$

The system $\mathbf{B}$ consists of the following operational rules:

\footnotetext{
${ }^{2}$ Meyer (2004) added in addition the axiom $T \rightarrow(A \rightarrow T)$ to the o-free axiomatization of the positive fragment of $\mathbf{B}$. The reason, presumably, being that the $\circ$-free fragment would otherwise be non-conservatively extended by $\circ$.

${ }^{3}$ If the structure to be replaced is clear from context, then I will on occasion drop the slashnotation to increase readability.
}

Australasian Journal of Logic (18:2) 2021, Article no. 2 


$$
\begin{aligned}
& \overline{A \triangleright A}^{(I d)} \\
& \frac{X ; A \triangleright B}{X \triangleright A \rightarrow B}{ }_{(\rightarrow I)} \quad \frac{X \triangleright A \rightarrow B \quad Y \triangleright A}{X ; Y \triangleright B}(\rightarrow E) \\
& \frac{X \triangleright A \quad Y \triangleright B}{X ; Y \triangleright A \circ B}(\circ I) \quad \frac{X \triangleright A \circ B \quad Y(A ; B) \triangleright C}{Y(A ; B / X) \triangleright C}(\circ E)
\end{aligned}
$$

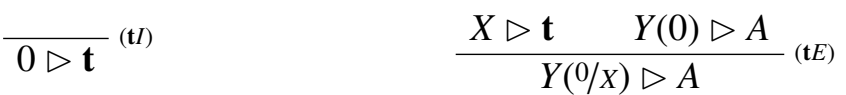

$$
\begin{aligned}
& \frac{X \triangleright A \quad X \triangleright B}{X \triangleright A \wedge B}(\wedge I) \quad \frac{X \triangleright A_{1} \wedge A_{2}}{X \triangleright A_{i}}\left(\wedge E_{i \leq 2}\right)
\end{aligned}
$$

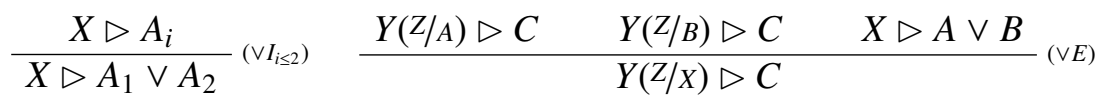

$$
\begin{aligned}
& \frac{X \triangleright \sim \sim A}{X \triangleright A}(\sim \sim E) \quad \frac{A \triangleright \sim B \quad X \triangleright B}{X \triangleright \sim A}(\sim I / \sim E) \\
& \frac{X \triangleright \perp}{X \triangleright A}\left(\perp E_{m}\right)
\end{aligned}
$$

I follow Restall in writing structural rules other than the cut rule on the form $X \Leftarrow X^{\prime}$. Any such rule is to be interpreted as the rule

$$
\frac{Y(X) \triangleright A}{Y\left(X^{\prime}\right) \triangleright A} \text {. }
$$

The structural rules of $\mathbf{B}$ are the following:

$$
\begin{aligned}
& \frac{X \triangleright A \quad Y(A) \triangleright B}{Y(A / X) \triangleright B}(\text { cut }) \\
& \text { (eB) } X,(Y, Z) \Leftarrow(X, Y), Z \\
& \text { (eCI) } \quad X, Y \Leftarrow Y, X \\
& (e W I) \quad X, X \Leftarrow X \\
& (e K) \quad X \Leftarrow X, Y \\
& \text { (Left Push) } \quad X \Leftarrow 0 ; X \\
& \text { (Left Pop) } \quad 0 ; X \Leftarrow X
\end{aligned}
$$

To distinguish Hilbert rules from consecution rules, I will on occasion call them, respectively, $H$-rules and c-rules.

Definition 5. (Proof) A proof of a consecution $X \triangleright A$ from a set of consecutions $\Gamma$ in the consecution system for $\mathbf{B}$ is defined to be a tree with $X \triangleright A$ as its root. Any node is a consecution which is either a member of $\Gamma$, and if so it is a leaf of the tree, or Australasian Journal of Logic (18:2) 2021, Article no. 2 
is obtained from immediately preceding nodes using a c-rule. The existential claim that there is such a proof will be written

$$
\Gamma \vdash_{\mathbf{B}}^{r} X \triangleright A .
$$

In the following we will need to combine proof-trees. Say, then, that $\Pi_{1}$ is the proof-tree below to the effect that $\{0 \triangleright A\} \vdash_{\mathbf{B}}^{r} 0 \triangleright A \vee C$, and $\Pi_{2}$ is the proof-tree below to the effect that $\{0 \triangleright B\} \vdash_{\mathbf{B}}^{r} 0 \triangleright(B \vee C) \wedge B$ :

$$
\frac{0 \triangleright A}{0 \triangleright A \vee C}\left(\vee I_{1}\right) \quad \frac{\frac{0 \triangleright B}{0 \triangleright B \vee C}\left(\vee I_{1}\right) \quad 0 \triangleright B}{0 \triangleright(B \vee C) \wedge B} \wedge I .
$$

If I then want to combine these two proof to obtain a proof that $\{0 \triangleright A, 0 \triangleright B\} r_{\mathbf{B}}^{r}$ $0 \triangleright(A \vee C) \wedge((B \vee C) \wedge B)$, I will simply write

$$
\frac{\Pi_{1} \quad \Pi_{2}}{0 \triangleright(A \vee C) \wedge((B \vee C) \wedge B)} \wedge I .
$$

If I want to show the premises or the conclusions of $\Pi_{1}$ and $\Pi_{2}$, I'll use a dotted line as follows:

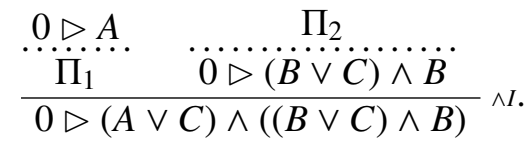

which, then, indicates that the premise-consecution $0 \triangleright A$ belongs to $\Pi_{1}$ and that $0 \triangleright(B \vee C) \wedge B$ is the conclusion of $\Pi_{2}$.

\section{Translations between calculi}

Restall proved in Restall (2000, ch. 4) what amounts to a weak soundness and completeness result, that is, he showed that a consecution $X \triangleright A$ is provable in his consecution calculus, for some logic $\mathbf{B}$, if and only if $\tau(X) \rightarrow A$ is a logical theorem in the Hilbert calculus for $\mathbf{B}$. $\tau$ is here a translation from structures and consecutions to formulas. Restall defines $\tau$ as follows:

Definition 6 (Translation of Consecution).

$$
\begin{aligned}
& \tau(A)=d_{f} A \quad \text { where } A \text { is any formula } \\
& \tau(0)={ }_{d f} \mathbf{t} \\
& \tau(X ; Y)={ }_{d f} \tau(X) \circ \tau(Y) \\
& \tau(X, Y)=d_{f f} \tau(X) \wedge \tau(Y) \\
& \tau(X \triangleright A)=_{d f} \tau(X) \rightarrow A \\
& \tau(\Gamma)=_{d f}\{\tau(z) \mid z \in \Gamma\} \text { where } \Gamma \text { is any set of structures or consecutions }
\end{aligned}
$$

Restall's soundness and completeness theorem, Restall (2000, Thm. 4.3), reads relative to $\mathbf{B}$ when translated into the jargon of this paper as follows:

$$
\varnothing \vdash_{\mathbf{B}}^{r}(X \triangleright A) \Longleftrightarrow \varnothing \vdash_{\mathbf{B}}^{h} \tau(X \triangleright A)
$$

Although Restall does consider what he calls proofs from assumptions (Restall, 2000 , p. 21) a notion which corresponds to proofs from non-empty sets of consecutions $\Gamma$, Restall's main focus, I think it is fair to say, is on proofs where the Australasian Journal of Logic (18:2) 2021, Article no. 2 
assumption set is empty. It is, then, the property $\left\{X \triangleright A \mid \varnothing \vdash_{\mathbf{L}}^{r} X \triangleright A\right\}$, for certain logics $\mathbf{L}$, which is Restall's main object of study, not the more general relation $\left\{\langle\Gamma, X \triangleright A\rangle \mid \Gamma \vdash_{\mathrm{L}}^{r} X \triangleright A\right\}$. The goal of this section is to show that Restall's result can be extended to a strong soundness and completeness result.

Lemma 2. For any structure $X$ :

$$
\varnothing \vdash_{\mathbf{B}}^{r} X \triangleright \tau(X)
$$

Proof. See Restall (2000, lem. 4.17).

Lemma 3. For any consecution $X \triangleright A$ :

$$
\{X \triangleright A\} \vdash_{\mathbf{B}}^{r} 0 \triangleright \tau(X \triangleright A) .
$$

Proof. Translating the second part of Restall (2000, lem. 4.17) into our jargon yields the statement $\varnothing \vdash_{\mathbf{B}}^{r} X \triangleright A \Longrightarrow \varnothing \vdash_{\mathbf{B}}^{r} \tau(X) \triangleright A$. Restall's proof, however suffices for deriving $\tau(X) \triangleright A$ from $X \triangleright A$, and so is a proof that $\{X \triangleright A\} \vdash_{\mathbf{B}}^{r} \tau(X) \triangleright A$. By using the Left Push rule and $(\rightarrow I)$ one then gets that $\{X \triangleright A\} \vdash_{\mathbf{B}}^{r} 0 \triangleright \tau(X) \rightarrow A$. The proof now ends since $\tau(X \triangleright A)=\tau(X) \rightarrow A$.

Lemma 4 (Soundness lemma). If $\Delta \Vdash A$ is a Hilbert-rule for $\boldsymbol{B}$, then for any set $\Gamma$, if $\Gamma \vdash_{\mathbf{B}}^{r} 0 \triangleright B_{i}$ for every $B_{i} \in \Delta$, then also $\Gamma \vdash_{\mathbf{B}}^{r} 0 \triangleright A$.

Proof. (R1): Assume that $\Pi_{1}$ is a proof that $\Gamma r_{\mathbf{B}}^{r} 0 \triangleright A \rightarrow B$ and $\Pi_{2}$ a proof that $\Gamma \vdash_{\mathbf{B}}^{r} 0 \triangleright A$.

$$
\frac{\ldots \ldots \Pi_{1} \ldots \ldots \quad \ldots \Pi_{2} \ldots}{\frac{0 ; 0 \triangleright B}{0 \triangleright B}{ }_{(L . P o p)}}(\rightarrow E)
$$

(R2): Assume that $\Pi_{1}$ is a proof that $\Gamma r_{\mathbf{B}}^{r} 0 \triangleright A$ and $\Pi_{2}$ a proof that $\Gamma r_{\mathbf{B}}^{r} 0 \triangleright B$.

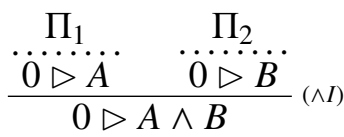

(R3): Assume that $\Pi_{1}$ is a proof that $\Gamma \vdash_{\mathbf{B}}^{r} 0 \triangleright A \rightarrow B$.

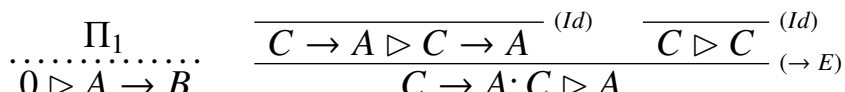

$$
\begin{aligned}
& \frac{\dddot{0 \triangleright} \rightarrow \rightarrow \rightarrow B}{0 ;(C \rightarrow A ; C) \triangleright B}{ }_{(L \text { Pop })}^{C} \rightarrow(\rightarrow E) \\
& C \rightarrow A ; C \triangleright B \quad \text { (L.Pop) } \\
& \overline{C \rightarrow A \triangleright C \rightarrow B}^{(\rightarrow I)} \\
& \frac{\frac{C \rightarrow A \rightarrow A \triangleright C \rightarrow B}{0}(\text { L.Push })}{0 \triangleright(C \rightarrow A) \rightarrow(C \rightarrow B)}(\rightarrow I)
\end{aligned}
$$

(R4)-(Rt): Left for the reader.

Theorem 1 (Strong soundness for B). For any set of consecutions $\Gamma$,

$$
\tau(\Gamma) \vdash_{\mathbf{B}}^{h} \tau(X \triangleright A) \Longrightarrow \Gamma \vdash_{\mathbf{B}}^{r} X \triangleright A
$$

Australasian Journal of Logic (18:2) 2021, Article no. 2 
Proof. Assume that $\tau(\Gamma) \vdash_{\mathbf{B}}^{h} \tau(X \triangleright A)$ and let $\alpha_{1}, \ldots, \alpha_{n}$ be a Hilbert proof of $\tau(X \triangleright A)$ from $\tau(\Gamma)$. The first goal is to prove that $\Gamma r_{\mathbf{B}}^{r} 0 \triangleright \alpha_{i}$ for every $i \leq n$. To that end: $\alpha_{i}$ is either a logical axiom of $\mathbf{B}$, a member of $\tau(\Gamma)$, or is obtained from prior $\alpha_{j}$ 's using a rule of $\mathbf{B}$. If $\alpha_{i}$ is an axiom of $\mathbf{B}$, then $\varnothing \vdash_{\mathbf{B}}^{h} \alpha_{i}$. Using (Rt) we then obtain that $\varnothing \vdash_{\mathbf{B}}^{h} \mathbf{t} \rightarrow \alpha_{i}$. Since $\tau\left(0 \triangleright \alpha_{i}\right)=\mathbf{t} \rightarrow \alpha_{i}$ we can use Restall's soundness theorem (Restall, 2000, thm. 4.16) to infer that $\varnothing \vdash_{\mathbf{B}}^{r} 0 \triangleright \alpha_{i}$. It then follows by the definition of a consecution-proof that $\Gamma r_{\mathbf{B}}^{r} 0 \triangleright \alpha_{i}$.

Assume that $\alpha_{i} \in \tau(\Gamma)$. Then $\alpha_{i}$ is the $\tau$-translation of some consecution $Y \triangleright C \in$ $\Gamma$. From Lem. 3 we have that $\{Y \triangleright C\} \vdash_{\mathbf{B}}^{r} 0 \triangleright \tau(Y \triangleright C)$, and so it follows that $\Gamma \vdash_{\mathbf{B}}^{r} 0 \triangleright \alpha_{i}$.

Assume that $\alpha_{i}$ is obtained using a rule of $\mathbf{B}$. We may then assume for induction that if $\Delta$ are the premises, then $\Gamma \vdash_{\mathbf{B}}^{r} 0 \triangleright \alpha_{j}$ for every $\alpha_{j} \in \Delta$. We can now use Lem. 4 to infer that $\Gamma \vdash_{\mathbf{B}}^{r} 0 \triangleright \alpha_{i}$.

Since $\alpha_{n}$ is $\tau(X \triangleright A)$, we can then infer that $\Gamma \vdash_{\mathbf{B}}^{r} 0 \triangleright \tau(X \triangleright A)$. By the definition of $\tau$ we can then infer that $\Gamma \vdash_{\mathbf{B}}^{r} 0 \triangleright \tau(X) \rightarrow A$. Let $\Pi_{1}$ be a proof of $0 \triangleright \tau(X) \rightarrow A$ from $\Gamma$. From Lem. 2 we have that $\varnothing \vdash_{\mathbf{B}}^{r} X \triangleright \tau(X)$. Let $\Pi_{2}$ be a proof of this. We then finally have that $\Gamma \vdash_{\mathbf{B}}^{r} X \triangleright A$ since we can combine and expand $\Pi_{1}$ and $\Pi_{2}$ as follows:

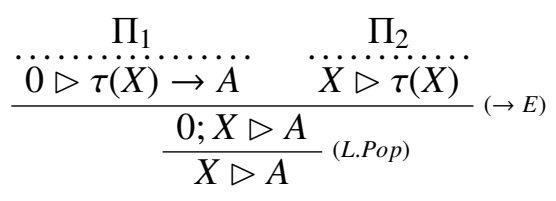

Lemma 5. $\tau(X(\tau(Y)))=\tau(X(Y))$

Proof. Left for the reader.

\section{Lemma 6.}

$$
\{A \rightarrow B\} \vdash_{\mathbf{B}}^{h} \tau(X(A)) \rightarrow \tau(X(A / B))
$$

Proof. See Restall (2000, lem. 4.19). ${ }^{4}$

Lemma 7 (Structural lemma). For every structural rule $X \Leftarrow X^{\prime}$ of $\boldsymbol{B}$,

$$
\varnothing \vdash_{\mathbf{B}}^{h} \tau\left(X^{\prime}\right) \rightarrow \tau(X)
$$

Proof. Left for the reader.

Lemma 8 (Distribution lemma).

$$
\varnothing \vdash_{\mathbf{B}}^{h} \tau(X(A \vee B)) \rightarrow \tau(X(A)) \vee \tau(X(B)) .
$$

Proof. See Restall (2000, lem. 4.20)

Lemma 9 (Completeness lemma). If

\footnotetext{
${ }^{4}$ Restall states his theorem as an admissible rule: "If there is a Hilbert proof of $A \rightarrow B$ then there is also a Hilbert proof of $\tau(X(A)) \rightarrow \tau(X(B))$." His proof, however, supports the stronger statement. Note, furthermore, that this lemma holds for every extension of $\mathbf{B}$ considered in this paper.
}

Australasian Journal of Logic (18:2) 2021, Article no. 2 


$$
\begin{array}{ccc}
X_{1} \triangleright A_{1} & \ldots & X_{n} \triangleright A_{n} \\
\hline Z \triangleright B
\end{array}
$$

is a consecution rule for $\boldsymbol{B}$, then for any set of consecutions $\Gamma$, if for every $i \leq n$, $\tau(\Gamma) \vdash_{\mathbf{B}}^{h} \tau\left(X_{i} \triangleright A_{i}\right)$, then also $\tau(\Gamma) \vdash_{\mathbf{B}}^{h} \tau(Z \triangleright B)$.

Proof. In the easiest case the rule has no premises, and so the conclusion is either $A \triangleright A$ or $0 \triangleright \mathbf{t}$. Since $\tau(A \triangleright A)=A \rightarrow A$ and $\tau(0 \triangleright \mathbf{t})=\mathbf{t} \rightarrow \mathbf{t}$, we do have that $\tau(\Gamma) \vdash_{\mathbf{B}}^{h} \tau(Z \triangleright B)$ for every set $\Gamma$.

Assume that $Z \triangleright B$ is obtained using a 1-premise operational rule. If it is $\left(\perp E_{m}\right)$, then we may assume for inductive hypothesis $(\mathrm{IH})$ that $\tau(\Gamma) \vdash_{\mathbf{B}}^{h} \tau(X \triangleright \perp)$. Since $\tau(X \triangleright \perp)=\tau(X) \rightarrow \perp$, we may use the axiom $\perp \rightarrow A$ together with the fact that $\rightarrow$ is transitive to infer that $\tau(\Gamma) \vdash_{\mathbf{B}}^{h} \tau(X) \rightarrow A$. Similar type of reasoning suffices in cases of the 1-premise rules $(\sim \sim E),\left(\vee I_{i \leq 2}\right)$, and $\left(\wedge E_{i \leq 2}\right)$. In the case of $(\rightarrow I)$, we can assume that $\tau(\Gamma) \vdash_{\mathbf{B}}^{h} \tau(X ; A \triangleright B)$. Since $\tau(X ; A \triangleright B)=\tau(X) \circ A \rightarrow B$, using the fusion rule (Ro) yields $\tau(\Gamma) \vdash_{\mathbf{B}}^{h} \tau(X) \rightarrow(A \rightarrow B)$, and since $\tau(X \triangleright A \rightarrow B)=$ $\tau(X) \rightarrow(A \rightarrow B)$, we're done.

Now to consider the 2-premise rules. In the case of $(\rightarrow E)$, the $(\mathrm{IH})$ gives us that $\tau(\Gamma) \vdash_{\mathbf{B}}^{h} \tau(X \triangleright A \rightarrow B)$ and $\tau(\Gamma) \vdash_{\mathbf{B}}^{h} \tau(Y \triangleright A)$. Using $\tau$, then, this translates to $\tau(\Gamma) \vdash_{\mathbf{B}}^{h} \tau(X) \rightarrow(A \rightarrow B)$ and $\tau(\Gamma) \vdash_{\mathbf{B}}^{h} \tau(Y) \rightarrow A$. Using (R4) and the transitivity of $\rightarrow$ we obtain that $\tau(\Gamma) \vdash_{\mathbf{B}}^{h} \tau(X) \rightarrow(\tau(Y) \rightarrow B)$, and so using the o-rule (Ro) we finally obtain that $\tau(\Gamma) \vdash_{\mathbf{B}}^{h} \tau(X) \circ \tau(Y) \rightarrow B$, and since $\tau(X ; Y \triangleright B)=\tau(X) \circ$ $\tau(Y) \rightarrow B$, we're done. $(\circ I),(\wedge I)$ and $(\sim I / \sim E)$ are all left to the reader. The last 2-premise operational rule is $(\circ E)$. (IH) gives us that $\tau(\Gamma) \vdash_{\mathbf{B}}^{h} \tau(X \triangleright A \circ B)$ and $\tau(\Gamma) \vdash_{\mathbf{B}}^{h} \tau(Y(A ; B) \triangleright C)$. Using $\tau$ we then have that $\tau(\Gamma) \vdash_{\mathbf{B}}^{h} \tau(X) \rightarrow A \circ B$ and, using Lem. 5, $\tau(\Gamma) \vdash_{\mathbf{B}}^{h} \tau(Y(A \circ B)) \rightarrow C$. Using Lem. 6 we obtain that $\tau(\Gamma) \vdash_{\mathbf{B}}^{h} \tau(Y(\tau(X))) \rightarrow \tau(Y(A \circ B))$ and so using Lem. 5 also that $\tau(\Gamma) \vdash_{\mathbf{B}}^{h} \tau(Y(X)) \rightarrow$ $\tau(Y(A \circ B))$. The transitivity of $\rightarrow$ they yields that $\tau(\Gamma) \vdash_{\mathbf{B}}^{h} \tau(Y(X)) \rightarrow C$ and since $\tau(Y(A ; B / X) \triangleright C)=\tau(Y(X)) \rightarrow C$, we're done.

The 3-premise operational rule $(\vee E)$ is proven in the same manner as $(\circ E)$ (using in addition Lem. 8) and is left for the reader.

For the cut-rule we get from (IH) that $\tau(\Gamma) \vdash_{\mathbf{B}}^{h} \tau(X \triangleright A)$ and $\tau(\Gamma) \vdash_{\mathbf{B}}^{h} \tau(Y(A) \triangleright B)$. Using $\tau$ this yields that $\tau(\Gamma) \vdash_{\mathbf{B}}^{h} \tau(X) \rightarrow A$ and $\tau(\Gamma) \vdash_{\mathbf{B}}^{h} \tau(Y(A)) \rightarrow B$. Using Lem. 5 and Lem. 6 we can then infer that $\tau(\Gamma) \vdash_{\mathbf{B}}^{h} \tau(Y(X)) \rightarrow \tau(Y(A))$ and so transitivity of $\rightarrow$ yields that $\tau(\Gamma) \vdash_{\mathbf{B}}^{h} \tau(Y(X)) \rightarrow B$. Since $\tau(Y(X) \triangleright B)=\tau(Y(X)) \rightarrow B$ we're done.

Last but not least we have the structural rules on the form

$$
\frac{Y(X) \triangleright A}{Y\left(X^{\prime}\right) \triangleright A} \text {. }
$$

From (IH) we may assume that $\tau(\Gamma) \vdash_{\mathbf{B}}^{h} \tau(Y(X) \triangleright A)$. For any structural rule $X \Leftarrow X^{\prime}$, Lem. 7 yields that $\varnothing \vdash_{\mathbf{B}}^{h} \tau\left(X^{\prime}\right) \rightarrow \tau(X)$, and so using Lem. 5 and Lem. 6 we get that $\varnothing \vdash_{\mathbf{B}}^{h} \tau\left(Y\left(X^{\prime}\right)\right) \rightarrow \tau(Y(X))$. Using $\tau$ and the transitivity of $\rightarrow$ then yields that $\tau(\Gamma) \vdash_{\mathbf{B}}^{h} \tau\left(Y\left(X^{\prime}\right) \triangleright A\right)$ which ends the proof

Australasian Journal of Logic (18:2) 2021, Article no. 2 
Theorem 2 (Strong Completeness for $\mathbf{B}$ ).

$$
\Gamma \vdash_{\mathbf{B}}^{r} X \triangleright A \Longrightarrow \tau(\Gamma) \vdash_{\mathbf{B}}^{h} \tau(X \triangleright A)
$$

Proof. As in the soundness case, the proof is a simple induction, this time to the effect that the $\tau$-translation of every consecution $Y \triangleright B$ occurring in the proof-tree for $\Gamma \vdash_{\mathbf{B}}^{r}(X \triangleright A)$ is Hilbert-derivable from $\tau(\Gamma)$. Every such consecution is either a member of $\Gamma$ or obtained from 0 or more consecutions using a rule. In the first case we have that $\tau(\Gamma) \vdash_{\mathbf{B}}^{h} \tau(X \triangleright A)$ since in this case $\tau(X \triangleright A) \in \tau(\Gamma)$. In the other case, Lem. 9 ensures that every consecution obtained using a rule is also Hilbert derivable from $\tau(\Gamma) .^{5}$

The soundness and completeness results show that every consecution derivation has a matching Hilbert derivation. However, they do not in themselves show the converse that every Hilbert derivation has a corresponding consecution derivation. This, however, is an easy corollary using the following translation from formulas to consecutions:

Definition 7.

$$
\begin{aligned}
& \iota(A)=d_{d f} \quad 0 \triangleright A \quad \text { where } A \text { is any formula } \\
& \iota(\Gamma)=_{d f}\{\iota(A) \mid A \in \Gamma\} \text { where } \Gamma \text { is any set of formulas }
\end{aligned}
$$

Corollary 1. For any set of formulas $\Gamma$ :

$$
\Gamma \vdash_{\mathbf{B}}^{h} A \Longleftrightarrow \iota(\Gamma) \vdash_{\mathbf{B}}^{r} \iota(A) .
$$

Proof. From the strong soundness and completeness theorems we have that

$$
\iota(\Gamma) \vdash_{\mathbf{B}}^{r} \iota(A) \Longleftrightarrow \tau(\iota(\Gamma)) \vdash_{\mathbf{B}}^{h} \tau(\iota(A))
$$

Now note that for any formula $A$ we have that $\tau(\iota(A))=\mathbf{t} \rightarrow A$ and similarly for sets of formulas. Using the $\mathbf{t}$-rule (Rt), the fact that $\mathbf{t}$ is an axiom of $\mathbf{B}$ and modus ponens, it is then easy to see that

$$
\tau(\iota(\Gamma)) \vdash_{\mathbf{B}}^{h} \tau(\iota(A)) \Longleftrightarrow \tau(\iota(\Gamma)) \vdash_{\mathbf{B}}^{h} A .
$$

Lastly, since $\Gamma \vdash_{\mathbf{B}}^{h} B$ for every $B \in \tau(\iota(\Gamma))$ and $\tau(\iota(\Gamma)) \vdash_{\mathbf{B}}^{h} B$ for every $B \in \Gamma$, we have that

$$
\tau(\iota(\Gamma)) \vdash_{\mathbf{B}}^{h} A \Longleftrightarrow \Gamma \vdash_{\mathbf{B}}^{h} A .
$$

This last corollary shows that what is sometimes called the external consequence relation (cf. Avron, 1988) of a sequent calculus- $\iota(\Gamma) \vdash_{\mathbf{B}}^{r} \iota(A)$ in this case-is in the case of $\mathbf{B}$ extensionally identical to the defined Hilbertian consequence relation $\vdash_{\mathbf{B}}^{h}$. The next section looks briefly at common ways of extending $\mathbf{B}$ to yield stronger logics, as well as on ways of expanding the set of connectives.

\footnotetext{
${ }^{5}$ To make this rigorous one could assign a depth-measure such that the conclusion of every rule is assign a depth one more than the maximum of its premises and then reformulated the proof as an induction on the depth of consecutions. I hope the reader will find the current proof satisfactory.

Australasian Journal of Logic (18:2) 2021, Article no. 2
} 


\section{Generalizing TO OTHER LOGICS}

We saw in the previous section that Restall's weak soundness and completeness result can be strengthened to a strong one in the case of $\mathbf{B}$. It is not only $\mathbf{B}$ which is strongly sound and complete, however; the result generalizes to all the systems of consecution logics that Restall considers. This section will give a glimpse at how.

4.1. A stronger falsum. Let's first look at the already mentioned stronger rule for $\perp$. Restall adds not $\left(\perp E_{m}\right)$, but rather: $\frac{X \triangleright \perp}{Y(X) \triangleright A}(\perp E)$. Starting from $\perp \triangleright \perp$ one can then use the rule to obtain $X ; \perp \triangleright \perp$, and therefore also $0 \triangleright C \rightarrow(\perp \rightarrow \perp)$ :

$$
\begin{gathered}
\frac{\perp \triangleright \perp}{C ; \perp{ }^{(I d)}}{ }^{(\perp E)} \\
\frac{\frac{D \triangleright \perp \rightarrow \perp}{0 ; C \triangleright \perp \rightarrow \perp}_{(L . P u s h)}^{(\rightarrow)}}{0 \triangleright C \rightarrow(\perp \rightarrow \perp)}(\rightarrow I)
\end{gathered}
$$

Let's define $\mathbf{B}^{\sharp \perp}$ to be $\mathbf{B}$ with $\left(\perp E_{m}\right)$ replaced by $(\perp E)$, and in the Hilbert case $\mathbf{B}$ with $C \rightarrow(\perp \rightarrow \perp)$ added as an extra axiom. What, then, about strong soundness and completeness for $\mathbf{B}^{\sharp \perp}$ ?

The soundness proof for $\mathbf{B}^{\sharp \perp}$ needs in addition to the one given for $\mathbf{B}$ only that $\varnothing r_{\mathbf{B}^{\sharp \perp}}^{r} 0 \triangleright C \rightarrow(\perp \rightarrow \perp)$, and this we have already seen to be the case.

Corollary 2 (Strong soundness for $\mathbf{B}^{\sharp \perp}$ ). For any set of consecutions $\Gamma$,

$$
\tau(\Gamma) \vdash_{\mathbf{B}^{\sharp \perp}}^{h} \tau(X \triangleright A) \Longrightarrow \Gamma \vdash_{\mathbf{B}^{\sharp \perp}}^{r} X \triangleright A
$$

The completeness proof is a bit more involved. We first need a lemma:

Lemma 10. $\varnothing r_{\mathbf{B}^{\sharp \perp}}^{h} \tau(Y(\perp)) \rightarrow \perp$

Proof. This is an inductive proof on the complexity of $\tau(Y(\perp))$. First note that ' $Y(\perp)$ ' indicates that $Y$ is a structure in which $\perp$ is a substructure, and so in the base case $Y(\perp)$ is simply $\perp$.

Assume, then, that $Y(\perp)$ is obtained from the structures $Z$ and $W$. In this case it follows that $\perp$ is a substructure of at least one of them, let's say $Z$. Thus we may for inductive hypothesis assume that $\varnothing \vdash_{\mathbf{B}^{\sharp}}^{h} \tau(Z(\perp)) \rightarrow \perp$, where ' $Z(\perp)$ ' simply indicates that $\perp$ is a substructure of $Z$. Now $Y(\perp)$ can be either of the following structures (the outermost parentheses are removed):

$$
\begin{array}{ll}
\text { (1) } Z(\perp), W & \text { (2) } W, Z(\perp) \\
\text { (3) } Z(\perp) ; W & \text { (4) } W ; Z(\perp)
\end{array}
$$

The $\tau$-translation of these structures are

$$
\begin{array}{lll}
\text { (1) } \tau(Z(\perp)) \wedge \tau(W) & \text { (2) } \tau(W) \wedge \tau(Z(\perp)) \\
\text { (3) } \tau(Z(\perp)) \circ \tau(W) & \text { (4) } \tau(W) \circ \tau(Z(\perp))
\end{array}
$$

Since both $\tau(Z(\perp)) \wedge \tau(W) \rightarrow \tau(Z(\perp))$ and $\tau(W) \wedge \tau(Z(\perp)) \rightarrow \tau(Z(\perp))$ are logical theorems of $\mathbf{B}^{\sharp \perp}$, and we have assumed that so is $\tau(Z(\perp)) \rightarrow \perp$, we have that $\varnothing r_{\mathbf{B}^{\sharp \perp}}^{h} \tau(Z(\perp)) \rightarrow \perp$ in both the first and the second case.

Australasian Journal of Logic (18:2) 2021, Article no. 2 
For (3) note that $\{A \rightarrow B\} \vdash_{\mathbf{B}^{\sharp \perp}}^{h} A \circ C \rightarrow B \circ C$ is a derivable rule of $\mathbf{B}^{\sharp \perp}$ (left for the reader). Thus from the assumption that $\varnothing r_{\mathbf{B}^{\sharp \perp}}^{h} \tau(Z(\perp)) \rightarrow \perp$, we obtain that $\varnothing \vdash_{\mathbf{B}^{\sharp \perp}}^{h} \tau(Z(\perp)) \circ \tau(W) \rightarrow \perp \circ \tau(W)$. Using $(\mathrm{A} \perp)$ and $(\mathrm{R} \circ)$ we get that $\perp \circ \tau(W) \rightarrow \perp$ is a logical theorem, and so we can infer that $\varnothing \vdash_{\mathbf{B}^{\sharp \perp}}^{h} \tau(Z(\perp)) \circ \tau(W) \rightarrow \perp$ using the transitivity of $\rightarrow$.

In the case of (4), note that $\{A \rightarrow B\} \vdash_{\mathbf{B}^{\sharp \perp}}^{h} C \circ A \rightarrow C \circ B$ is also a derivable rule of $\mathbf{B}^{\sharp \perp}$. Thus from the assumption that $\varnothing \vdash_{\mathbf{B}^{\sharp \perp}}^{h} \tau(Z(\perp)) \rightarrow \perp, \varnothing \vdash_{\mathbf{B}^{\sharp \perp}}^{h} \tau(W) \circ \tau(Z(\perp)) \rightarrow$ $\tau(W) \circ \perp$. Since $\tau(W) \rightarrow(\perp \rightarrow \perp)$ is an axiom of $\mathbf{B}^{\sharp \perp}$, using (Ro) once yields that $\varnothing \vdash_{\mathbf{B}^{\sharp} \perp}^{h} \tau(W) \circ \tau(Z(\perp)) \rightarrow \perp$, which, then, completes the proof.

Corollary 3 (Strong completeness for $\mathbf{B}^{\sharp \perp}$ ). For any set of consecutions $\Gamma$,

$$
\Gamma \vdash_{\mathbf{B}^{\sharp \perp}}^{r}(X \triangleright A) \Longrightarrow \tau(\Gamma) \vdash_{\mathbf{B}^{\sharp \perp}}^{h} \tau(X \triangleright A)
$$

Proof. The proof of Thm. 2 carries over to $\mathbf{B}^{\sharp \perp}$ if we can show that Completeness lemma-Lem. 9-can be extended so as to also cover $(\perp E)$. To that end we may assume for inductive hypothesis that $\tau(\Gamma) \vdash_{\mathbf{B}^{\sharp \perp}}^{h} \tau(X \triangleright \perp)$. The goal is to show that $\tau(\Gamma) \vdash_{\mathbf{B}^{\sharp \perp}}^{h} \tau(Y(X) \triangleright \perp)$. First of all we have that $\tau(X \triangleright \perp)=\tau(X) \rightarrow \perp$. Using Lem. 5 and Lem. 6 we have that $\tau(\Gamma) \vdash_{\mathbf{B}^{\sharp \perp}}^{h} \tau(Y(X)) \rightarrow \tau(Y(\perp))$. From Lem. 10 we have that $\varnothing \vdash_{\mathbf{B}^{\sharp} \perp}^{h} \tau(Y(\perp)) \rightarrow \perp$. Using this together with $(\mathrm{A} \perp)$ and the transitivity of $\rightarrow$, we then obtain that $\tau(\Gamma) \vdash_{\mathbf{B}^{\sharp \perp}}^{h} \tau(Y(X)) \rightarrow A$, and therefore $\tau(\Gamma) \vdash_{\mathbf{B}^{\sharp \perp}}^{h} \tau(Y(X) \triangleright A)$.

4.2. A stronger negation. The logic $\mathbf{D W}$ is in the Hilbertian case obtained by replacing the contraposition rule (R5) by its axiomatic version $(A \rightarrow \sim B) \rightarrow(B \rightarrow$ $\sim A$ ). DW is not only stronger than $\mathbf{B}$, but also stronger than $\mathbf{B}^{\sharp \perp}$ as the following derivation shows:

Lemma 11. $\varnothing \vdash_{\mathrm{DW}}^{h} C \rightarrow(\perp \rightarrow \perp)$.
(1) $\perp \rightarrow \sim(C \circ \sim \perp)$
$A \perp$
(2) $C \circ \sim \perp \rightarrow \sim \perp$
$1, R 5$
(3) $C \rightarrow(\sim \perp \rightarrow \sim \perp)$
2, Lem. 1
(4) $(\sim \perp \rightarrow \sim \perp) \rightarrow(\perp \rightarrow \sim \sim \perp)$ A8
(5) $C \rightarrow(\perp \rightarrow \sim \sim \perp)$
3, 4, transitivity
(6) $(C \circ \perp) \rightarrow \sim \sim \perp$
$5, R \circ$
(7) $\sim \sim \perp \rightarrow \perp$
A7
(8) $(C \circ \perp) \rightarrow \perp$
6, 7, transitivity
(9) $C \rightarrow(\perp \rightarrow \perp)$
8, Lem. 1

The negation rules of $\mathbf{B}-(\sim \sim E)$ and $(\sim I / \sim E)$-yield what Restall calls a de Morgan negation. The consecution calculus for $\mathbf{D W}$ is obtained by replacing such a negation with its strict variant; by replacing $(\sim I / \sim E)$ with its semicolon variant:

$$
\frac{X ; A \triangleright \sim B \quad Y \triangleright B}{X ; Y \triangleright \sim A}(\sim I ; \sim E)
$$

Australasian Journal of Logic (18:2) 2021, Article no. 2 


\begin{tabular}{|l|l|l|}
\hline Hilbert axiom & label & structural rule \\
\hline$(A \rightarrow B) \rightarrow((C \rightarrow A) \rightarrow(C \rightarrow B))$ & $\mathrm{B}$ & $X ;(Y ; Z) \Leftarrow(X ; Y) ; Z$ \\
$(A \rightarrow B) \rightarrow((B \rightarrow C) \rightarrow(A \rightarrow C))$ & $\mathrm{B}^{\prime}$ & $X ;(Y ; Z) \Leftarrow(Y ; X) ; Z$ \\
$A \rightarrow((A \rightarrow B) \rightarrow B)$ & $\mathrm{CI}$ & $X ; Y \Leftarrow Y ; X$ \\
$A \wedge(A \rightarrow B) \rightarrow B$ & WI & $X ; X \Leftarrow X$ \\
$A \rightarrow(B \rightarrow A)$ & $\mathrm{K}$ & $X \Leftarrow X ; Y$ \\
\hline
\end{tabular}

TABLE 1. Some structural rules and their corresponding axioms

Strong soundness and completeness carries easily over to DW; details are left for the reader.

4.3. Adding structural rules. Well-known relevant logics such as $\mathbf{T W}, \mathbf{R W}$ and $\mathbf{R}$, as well as the non-relevant logic RWK, are obtained by adding axioms in the case of the Hilbert calculus, and more structural rules in the case of the consecution calculus. These four logics are obtained using the axioms and rules on display in Table. 1 as follows:

- TW: obtained from DW by adding B and B' from Table. 1. (R3) and (R4) can be deleted from the Hilbert calculus.

- RW: obtained from TW adding CI from Table. 1. o can be defined using $A \circ B={ }_{d f} \sim(A \rightarrow \sim B)$, and so (A०) and (R०) can be deleted from the Hilbert calculus. (Rt) can be replaced by the axiom $A \rightarrow(\mathbf{t} \rightarrow A)$.

- R: obtained from $\mathbf{R W}$ by adding WI.

- RWK: obtained from $\mathbf{R W}$ by adding K. Axioms for $\mathbf{t}$ and $\perp$ can be deleted as $\mathbf{t}$ can be defined as $p \rightarrow p$ some some $p$, and $\perp$ as $\sim \mathbf{t}$.

In all these cases it is fairly easy to modify the strong soundness and completeness proof seeing as for every structural rule $X \Leftarrow X^{\prime}$ of $\mathbf{L} \in\{\mathbf{T W}, \mathbf{R W}, \mathbf{R}, \mathbf{R W K}\}$, it is the case that

$$
\varnothing \vdash_{\mathbf{L}}^{h} \tau\left(X^{\prime}\right) \rightarrow \tau(X)
$$

Details are left for the reader.

4.4. More connectives. Negation is in Restall (2000) considered as belonging to the class of modalities, and more precisely to the class of negative modalities. The consecution rules for ' $\sim$ ' presented here are only but a few of the negation principles considered by Restall. Restall also considers positive modalities of the more familiar sort, as well as non-normal modalities-both the so-called Kleene star operator '*, ' as well as Girard's exponential operator '!.' It will not be possible to consider them all in this paper, although it should be noted that each of them can easily be shown to survive a strong soundness and completeness proof using the same basic technique as presented in this paper.

The left-to-right conditional , ' $\rightarrow$,' left-residuates the fusion connective in that sense that the left residuation rules

$$
\{A \circ B \rightarrow C\} \Vdash A \rightarrow(B \rightarrow C) \quad\{A \rightarrow(B \rightarrow C)\} \Vdash A \circ B \rightarrow C
$$

Australasian Journal of Logic (18:2) 2021, Article no. 2 
hold. For logics in which permutation holds-logics like RW-there is only one way to define a residual of $\circ$. In logics without full permutation there is yet another way, however, namely as the converse right-to-left conditional ' $\leftarrow$ ' which rightresiduates the fusion connective in that the Hilbert rules

$$
\{A \circ B \rightarrow C\} \Vdash B \rightarrow(C \leftarrow A) \quad\{B \rightarrow(C \leftarrow A)\} \Vdash A \circ B \rightarrow C
$$

hold. Relevant logics are usually defined without such a converse conditional, but related logics sometimes are. ${ }^{6}$ Restall's stated consecutions rules for ' $\leftarrow$ ' are as follows:

$$
\frac{A ; X \triangleright B}{X \triangleright B \leftarrow A}(\leftarrow I) \frac{X \triangleright B \leftarrow A \quad Y \triangleright A}{Y ; X \triangleright B}(\leftarrow E)
$$

The strong soundness and completeness results easily extend to logics outfitted with the right-to-left conditional with $(\leftarrow I)$ and $(\leftarrow E)$ added to the consecution calculus and the two mentioned Hilbert rules to the corresponding Hilbert calculus. I leave it to the reader to verify the details. It should be noted, however, that one can derive Restall's stronger $\perp$-rule, $(\perp E)$, using the weaker $\left(\perp E_{m}\right)$-rule together with the converse conditional. This conditional, therefore, cannot be added conservatively to weak logics such as $\mathbf{B}$ as it yields $C \rightarrow(\perp \rightarrow \perp)$ as a logical theorem. $^{78}$

\section{Generalizing fURTHer: AdDING META-INFERENCES - THE CASE OF REASONING BY}

CASES IN SUBSTRUCTURAL PROOF THEORY

Reasoning by cases is the metainferential rule that allows one to infer that a proposition follows from a disjunction provided it follows from both disjuncts. The sense in which it is metainferential is that it relates claims of following from to other such claims. As such it is usually thought of as a statement about a consequence relation, and not as a proper rule.

In the Hilbertian case, reasoning by cases holds for a logic $\mathbf{L}$ just in case $r_{\mathbf{L}}^{h}$ is closed under the rule ${ }^{9}$

$$
\frac{\{A\} \vdash_{\mathbf{L}}^{h} C \quad\{B\} \vdash_{\mathbf{L}}^{h} C}{\{A \vee B\} \vdash_{\mathbf{L}}^{h} C}(\mathrm{H}-\mathrm{RbC})
$$

Restall—see Restall (1994, thm. 5.2)—gave a nifty proof in that any logic L extending DW and which has only (R1) and (R2) as primitive rules is closed under the meta-rule of reasoning by cases. For other logics-logics like $\mathbf{B}, \mathbf{D W}$ and

\footnotetext{
${ }^{6}$ The most prominent example being Labek-type logics (cf. Lambek, 1958).

${ }^{7}$ A simple Hilbert proof goes as follows: $\perp \rightarrow(\perp \leftarrow C)$ is an instance of $(A \perp)$, and so the rightmost version of the right residuation rules yields $C \circ \perp \rightarrow \perp$ from which the leftmost version of the left residuation rules yields the sought-after conclusion. A similar proof using the consecution calculus can easily be found; I leave it for the reader. John Slaney's computer program MaGIC (Slaney, 1995) can easily be used to find a counter-model to $C \rightarrow(\perp \rightarrow \perp)$ for the logic $\mathbf{B}$.

${ }^{8}$ I would like to thank one of the referees for pressing the issue of the converse conditional, as well as pointing out its connection to Restall's stronger $\perp$-rule.

${ }^{9}$ An equivalent way to state $(\mathrm{RbC})$ is that $\vdash_{\mathbf{L}}^{h}$ be closed under the meta-rule that $\{A \vee C\} \vdash_{\mathbf{L}}^{h} B \vee C$ if $\{A\} \vdash_{\mathbf{L}}^{h} B$.
}

Australasian Journal of Logic (18:2) 2021, Article no. 2 
TW-it can be shown that $(\mathrm{H}-\mathrm{RbC})$ does not hold. For instance in all these logics it is the case that $\{A \wedge(A \rightarrow B)\} \vdash_{\mathbf{L}}^{h} B \vee C$ and $\{C\} \vdash_{\mathbf{L}}^{h} B \vee C$, but not that $\{(A \wedge(A \rightarrow B)) \vee C\} \vdash_{\mathbf{L}}^{h} B \vee C .{ }^{10}$ Note, furthermore, that even though (H-RbC) holds for logics like RW, RWK and $\mathbf{R}$, the result does not necessarily hold if the logic in question is extended by more primitive rules. For this reason it is worth considering how to ensure that $(\mathrm{H}-\mathrm{RbC})$ holds. One way to do so is to add disjunctive rules: for every primitive rule $\left\{A_{1}, \ldots, A_{n}\right\} \Vdash B$, to add in addition the rule $\left\{A_{1} \vee C, \ldots, A_{n} \vee C\right\} \Vdash B \vee C$. For a proof that this suffices for (H-RbC), see Priest and Sylvan (1992, p. 219). Another way is to add meta-rules as primitive rules. This was, to my knowledge, first done in Brady (1984). Another way is to revise the definition of a Hilbert proof. This is the approach of Øgaard (2017) where a Hilbert proof is defined to be a nested list so as to allow for subproofs. These approaches can be proven to be equivalent, but since the disjunctive-rulesapproach requires the least modification to the current set-up, I'll stick to it.

Definition 8. The relation $\vdash_{\mathbf{L}^{d}}^{h}$ is obtained from $\vdash_{\mathbf{L}}^{h}$ by adding every disjunctive version of every primitive rule of $\boldsymbol{L}$.

The consecution version of the external version of reasoning by cases is readily seen to be the following rule:

$$
\begin{array}{ccc}
{[0 \triangleright A]} & {[0 \triangleright B]} \\
\vdots & \vdots & \\
0 \triangleright C & 0 \triangleright C & 0 \triangleright A \vee B \\
\hline & 0 \triangleright C-R b C)
\end{array}
$$

The bracketed consecutions are intended as "temporary" assumptions which are then discharged by the $(C-R b C)$-rule. To allow for this we must update the definition of a proof-tree to allow in addition any temporary assumption $[X \triangleright A]$, but requiring that the tree does not contain any such assumptions which are not discharged.

Definition 9. The relation $\vdash_{\mathbf{L}^{d}}^{r}$ is obtained from $\vdash_{\mathbf{L}}^{r}$ by adding $(C-R b C)$ to the stock of rules of $\boldsymbol{L}$.

There are two complicating factors that need to be accounted for: the number of times a temporary assumption can be stated, and that sub-proofs from temporary assumptions may occur within other such sub-proofs. I'll comment one these in turn.

One of the main motivating reasons for using trees as proof structures is that they allow rules to only apply to premises immediately preceding the conclusion. Thus a premise can only be used once. Say, then, that one wants to prove the consecution $A \wedge(B \wedge C) \triangleright(A \wedge B) \wedge C$. One proof-tree showing that this is indeed

\footnotetext{
${ }^{10}$ Slaney's MaGIC can also in this case be used to find a counter-model. Australasian Journal of Logic (18:2) 2021, Article no. 2
} 
a provable consecution is the following:

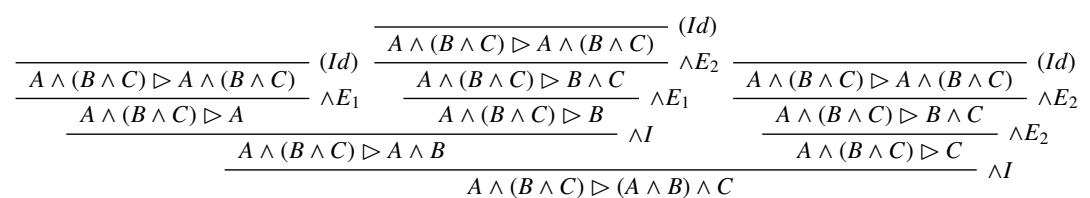

All the three branch in this tree has an identical initial segment. Since we can freely start a new branch with an instance of the (Id)-rule, or by any consecution occurring in the premise set, this kind of multiplying is innocuous. However, temporary assumptions need to be treated in the same kind of manner, and so a more representative presentation of the reasoning by cases rule is rather as follows:

$$
\begin{array}{ccc}
{[0 \triangleright A] \ldots[0 \triangleright A]} & {[0 \triangleright B] \ldots[0 \triangleright B]} & \\
\ddots \vdots \dot{C}^{\cdot} & \ddots \dot{.^{*}} & \\
0 \triangleright C & 0 \triangleright C & 0 \triangleright A \vee B \\
\hline & 0 \triangleright C
\end{array}
$$

An application of the $(C-R b C)$-rule is then understood to discharge every occurrence of the temporary assumptions.

The other complicating factor is that we need to allow for nested uses of reasoning by cases. As an easy example using a Fitch-style proof system, consider the following proof of $\left(A_{1} \wedge A_{2}\right) \vee C$ from $\left(A_{1} \vee C\right) \wedge\left(A_{2} \vee C\right)$ :

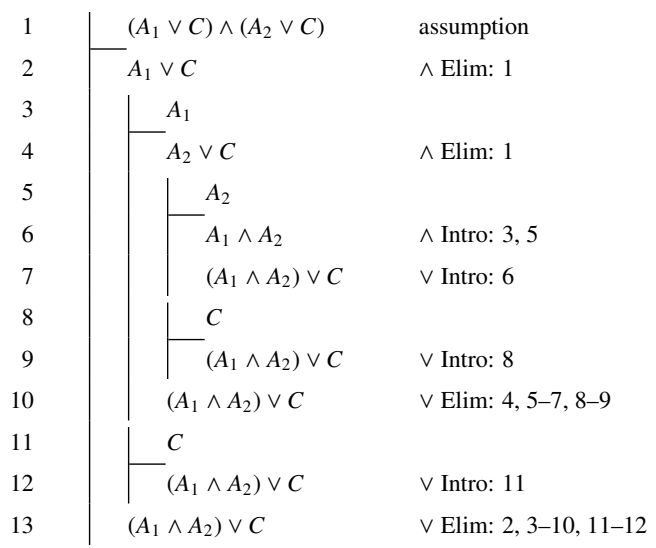

Regrettably, the proof to the same effect in the consecution calculus extended with $(C-R b C)$ is not nearly as neat as the Fitch-style proof as the proof of the following lemma shows:

Lemma 12. $\left\{0 \triangleright\left(A_{1} \vee C\right) \wedge\left(A_{2} \vee C\right)\right\} \vdash_{\mathbf{B}^{d}}^{r} 0 \triangleright\left(A_{1} \wedge A_{2}\right) \vee C$

Proof. Let in the following $\alpha=d_{d f}\left(A_{1} \vee C\right) \wedge\left(A_{2} \vee C\right)$ and $\beta={ }_{d f}\left(A_{1} \wedge A_{2}\right) \vee C$. I have decorated the proof with indices to show which temporary assumptions are discharged by which instance of $(C-R b C) .{ }^{11}$

\footnotetext{
${ }^{11}$ Note that we do not need $(C-R b C)$ to prove this seeing as the fact that $\varnothing \vdash_{\mathbf{B}}^{h}\left(A_{1} \vee C\right) \wedge\left(A_{2} \vee\right.$ $C) \triangleright\left(A_{1} \wedge A_{2}\right) \vee C$. However, if we use this, then we need to apply the cut-rule.
} 


$$
\begin{aligned}
& \frac{\left[0 \triangleright A_{1}\right]_{1} \quad\left[0 \triangleright A_{2}\right]_{2}}{0 \triangleright A_{1} \wedge A_{2}}\left(\vee I_{1}\right) \frac{[0 \triangleright I)}{\frac{0 \triangleright \beta}{0 \triangleright \beta}\left(\vee I_{2}\right) \frac{0 \triangleright \alpha}{0 \triangleright A_{1} \vee C}\left(\wedge E_{1}\right)}(C-R b C)_{2,3} \frac{[0 \triangleright C]_{4}}{0 \triangleright \beta}\left(\vee I_{2}\right) \\
& \frac{0 \triangleright \beta}{0 \triangleright-R b C)_{1,4}}
\end{aligned}
$$

The proof of the above lemma shows that even though an application of $(C-R b C)$ cancels temporary assumptions, that cannot be understood as cancelling the whole sub-branch which makes up the sub-proof as it might be entangled in yet another sub-proof-this happens in the above proof where $\left[0 \triangleright A_{1}\right]_{1}$ must be available for use in the last application of $(C-R b C)$ and so cannot be cancelled by the above application of the rule. The final update, then, is that $(C-R b C)$ is to be taken as cancelling at least one instance of each of the temporary assumptions, and to classify as a proper proof, the tree as a whole cannot have undischarged temporary assumptions.

Lemma 13 (Soundness lemma for $\mathbf{B}^{d}$ ). If $\Delta \Vdash A$ is a Hilbert-rule for $\mathbf{B}^{d}$, then for any set $\Gamma$, if $\Gamma r_{\mathbf{B}^{d}}^{r} 0 \triangleright B_{i}$ for every $B_{i} \in \Delta$, then also $\Gamma r_{\mathbf{B}^{d}}^{r} 0 \triangleright A$.

Proof. We simply update the soundness lemma for $\mathbf{B}$ - Lem. 4-so as also to cover the disjunctive rules of $\mathbf{B}^{d}$. $\mathbf{B}$ only has Hilbert rules with at most two premises, so any disjunctive rule of $\mathbf{B}^{d}$ is either on the form $\left\{A_{1} \vee C\right\} \Vdash B \vee C$ or on the form $\left\{A_{1} \vee C, A_{2} \vee C\right\} \Vdash B \vee C$. Lem. 4 shows that for every primitive rule $\left\{A_{1}, A_{2}\right\} \Vdash B$ of $\mathbf{B}$, that for any set of consecutions $\Theta$, if $\Pi_{i}$ is a proof of $0 \triangleright A_{i}$ from $\Theta$, then the $\Pi_{i}$ 's can be combined and expanded into a proof $\Pi$ of $0 \triangleright B$. We now reuse this to also cover the disjunctive rules.

If the rule in question is $\left\{A_{1} \vee C\right\} \Vdash B \vee C$, we can use $\Pi$ and $(C-R b C)$ to show that any proof $\Sigma_{1}$ that $\Gamma \vdash_{\mathbf{B}^{d}}^{r} 0 \triangleright A_{1} \vee C$ can be expanded to a proof that $\Gamma r_{\mathbf{B}^{d}}^{r} 0 \triangleright B \vee C$ :

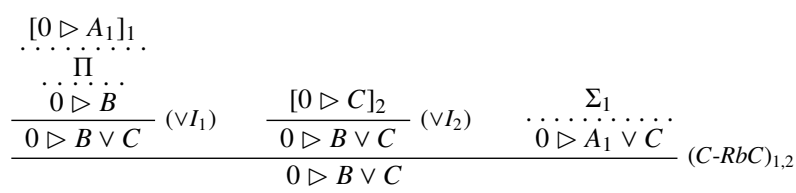

The case where the rule has two premises is handled in the same way, where now $\Sigma_{i}$ is a proof that $\Gamma r_{\mathbf{B}^{d}}^{r} 0 \triangleright A_{i} \vee C, \Psi$ is the proof from Lem. 12, and $\Pi$ is the proof of $0 \triangleright B$ obtained from proofs with conclusions $0 \triangleright A_{1}$ and $0 \triangleright A_{2}$ (with premises from some possibly $\Gamma$-distinct set $\Theta$ ). The resultant tree looks as follows:

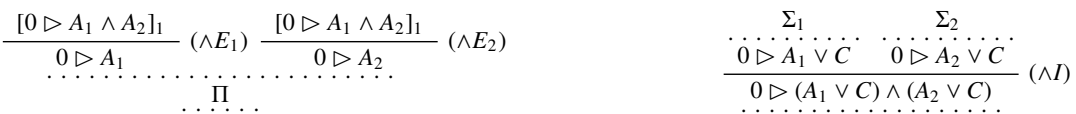

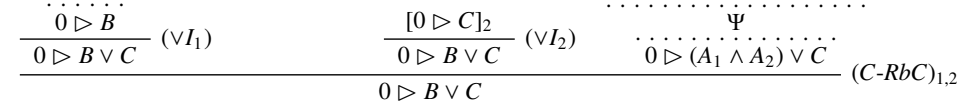

Australasian Journal of Logic (18:2) 2021, Article no. 2 
Theorem 3 (Strong soundness for $\mathbf{B}^{d}$ ). For any set of consecutions $\Gamma$,

$$
\tau(\Gamma) \vdash_{\mathbf{B}^{d}}^{h} \tau(X \triangleright A) \Longrightarrow \Gamma \vdash_{\mathbf{B}^{d}}^{r} X \triangleright A
$$

Proof. The proof is the same as that of Thm. 1

Lemma 14 (Completeness lemma for $\left.\mathbf{B}^{d}\right)$.

- If $\frac{X_{1} \triangleright A_{1} \quad \ldots \quad X_{n} \triangleright A_{n}}{Z \triangleright B}$ is a consecution rule for $\boldsymbol{B}$ other than $(C-R b C)$, then for any set of consecutions $\Gamma$, if for every $i \leq n, \tau(\Gamma) \vdash_{\mathbf{B}}^{h}$ $\tau\left(X_{i} \triangleright A_{i}\right)$, then also $\tau(\Gamma) \vdash_{\mathbf{B}}^{h} \tau(Z \triangleright B)$.

- If $0 \triangleright C$ is the conclusion of $(C-R b C)$ with major premise $0 \triangleright A \vee B$, then if all of

$$
\begin{array}{rll}
\tau(\Gamma) & \vdash_{\mathbf{B}}^{h} & \tau(0 \triangleright A \vee B) \\
\tau(\Gamma) \cup \tau\left(\Delta_{1}\right) \cup\{\tau(0 \triangleright A)\} & \vdash_{\mathbf{B}}^{h} \tau(0 \triangleright C) \\
\tau(\Gamma) \cup \tau\left(\Delta_{2}\right) \cup\{\tau(0 \triangleright B)\} & \vdash_{\mathbf{B}}^{h} \tau(0 \triangleright C)
\end{array}
$$

hold, where $\Delta_{1}\left(\Delta_{2}\right)$ is the set of temporary assumptions in the sub-proof starting with $[0 \triangleright A]([0 \triangleright B])$ that remains undischarged after the application of $(C-R b C)$, then also

$$
\tau(\Gamma) \cup \tau\left(\Delta_{1}\right) \cup \tau\left(\Delta_{2}\right) \vdash_{\mathbf{B}}^{h} \tau(0 \triangleright C) .
$$

Proof. This follows from the completeness lemma for B (Lem. 9) by noting that not only does $(\mathrm{H}-\mathrm{RbC})$ hold for $\vdash_{\mathbf{B}^{d}}^{h}$, but also the generalized version of it that for any set of formulas $\Xi_{1}, \Xi_{2}$ and $\Pi$,

$$
\frac{\Pi \cup \Xi_{1} \cup\{A\} \vdash_{\mathbf{L}}^{h} C \quad \Pi \cup \Xi_{2} \cup\{B\} \vdash_{\mathbf{L}}^{h} C}{\Pi \cup \Xi_{1} \cup \Xi_{2} \cup\{A \vee B\} \vdash_{\mathbf{L}}^{h} C}(\mathrm{gH}-\mathrm{RbC}) .
$$

I leave it to the reader to verify this.

Theorem 4 (Strong Completeness for $\mathbf{B}^{d}$ ).

$$
\Gamma \vdash_{\mathbf{B}^{d}}^{r} X \triangleright A \Longrightarrow \tau(\Gamma) \vdash_{\mathbf{B}^{d}}^{h} \tau(X \triangleright A)
$$

Proof. The proof is similar to that of Lem. 9, but instead of proving $\tau(\Gamma) \vdash_{\mathbf{B}}^{h}$ $\tau(Y \triangleright B)$ for every consecution $Y \triangleright B$ occurring in the proof-tree, we prove rather $\tau(\Gamma) \cup \tau\left(\Delta_{Y \triangleright B}\right) \vdash_{\mathbf{B}^{d}}^{h} \tau(Y \triangleright B)$, where $\Delta_{Y \triangleright B}$ is the set of undischarged consecutions occurring on some branch above $Y \triangleright B$. Then since every temporary assumption is eventually discharged, $\tau(\Gamma) \vdash_{\mathbf{B}^{d}}^{h} \tau(X \triangleright A)$. I leave it to the reader to complete the proof.

Corollary 4. For any set of formulas $\Gamma$ :

$$
\Gamma r_{\mathbf{B}^{d}}^{h} A \Longleftrightarrow \iota(\Gamma) r_{\mathbf{B}^{d}}^{r} \iota(A) .
$$

Proof. The proof is identical to that of Cor. 1 .

Australasian Journal of Logic (18:2) 2021, Article no. 2 
5.1. Other meta-rules. It would be possible to consider adding other meta-rules. When extending the logic to include quantifiers, for instance, the quantified version of reasoning by cases, namely

$$
\text { (M2) } \frac{\Gamma, A(x / y) \vdash_{\mathbf{L}}^{h} B}{\Gamma, \exists x A \vdash_{\mathbf{L}}^{h} B} y \notin F V(\Gamma \cup\{\exists x A, B\})
$$

would be a prime candidate. Another possible meta-rule extension is possible in the context of the $\omega$ rule

$$
(\mathrm{R} \omega) \quad A(0), \ldots, A(\bar{n}), \ldots \Vdash \forall x A(x)
$$

It seems that if one for every natural number $n$ can infer $B$ from $A(\bar{n})$ together with premises $\Gamma$, then $B$ ought to follow also from $\Gamma$ together with the assumption that there is some number which is $A$. This is codified in the following meta-rule:

$$
\left(\mathrm{M} 2_{\omega}\right) \frac{\Gamma, A(0) \vdash_{\mathbf{L}}^{h_{\omega}} B}{\Gamma, \exists x A \vdash_{\mathbf{L}}^{h_{\omega}} B}
$$

Consecution meta-rules for these could be found. However, going further in depth into these matters is beyond the scope of this paper.

\section{GENERALIZING FURTHER STILL: ADDING THEORY-DEPENDENT RULES}

For logics in the vicinity of relevant logics it is generally the case that adding an axiom $A \rightarrow B$ is stronger than adding the Hilbert-rule $\{A\} \Vdash B$. But seeing as axioms can be viewed as 0-premise rules, it is only natural to consider theories made up from not only axioms, but from rules as well. I propose to call such rules theory-dependent rules. ${ }^{12}$

One such theory-dependent rule is the $\omega$-rule encountered in the previous section. This rule is sometimes added to replace the induction axiom of Peano arithmetic in both classical as well as non-classical logics. Meyer pioneered the study of relevant arithmetic. Meyer used the usual Hilbert-type consequence relation$P A \vdash_{\mathbf{L}}^{h} A$ where $P A$ is the set of Peano axioms-when investigating relevant arithmetic. In some writings, however, Meyer used the induction axiom $A(0) \wedge$ $\forall x\left(A(x) \rightarrow A\left(x^{\prime}\right)\right) \rightarrow \forall x A(x)$, whereas he in other preferred a rule version of induction. $^{13}$

A natural way to generalize the consequence relation $r_{\mathbf{L}}^{h}$ to also cover theorydependent rules is simply to allow premise-sets to be set of pairs $\langle\Delta, A\rangle$, where

\footnotetext{
${ }^{12}$ The notion of a theory-dependent rule is but one of many ways the general notion of a rule can be precisified. See Brady $(1993,1994)$ for other interesting notions of rules in the context of relevant logics.

${ }^{13}$ For an example of the first approach, see Friedman and Meyer (1992). For the second, see Meyer (1976) where he even writes that "[n]ot all forms of the mathematical induction principle are relevantly valid. A sufficient form of this principle, however, is the rule $R M I$ : 'From $A 0$ and $A x \rightarrow A x^{\prime}$, infer $A x$.' "' (Meyer, 1976, p. 133). For arithmetics over other logics where induction is stated as a rule instead of as an axiom, see Meyer and Mortensen (1984), Meyer and Restall (1996), Meyer and Restall (1999), Restall (1992, 2010). I should also note that Dunn has investigated Robinson's arithmetic using R (Dunn, 1979).

Australasian Journal of Logic (18:2) 2021, Article no. 2
} 
$\Delta \Vdash A$ is the theory-dependent rule added, and if $A$ is to be an axiom, then $\Delta=\varnothing$. Just as we have seen that for any set of formulas $\Theta, \Theta \vdash_{\mathbf{B}}^{h} A \Longleftrightarrow \iota(\Theta) \vdash_{\mathbf{B}}^{r} \iota(A)$, it should be clear by now that for any set of pair-premises $\Lambda, \Lambda \vdash_{\mathbf{B}}^{h} A$ if and only if the consecution $0 \triangleright A$ is derivable from the set $\{0 \triangleright B \mid\langle\varnothing, B\rangle \in \Lambda\}$ and where for every $\langle\Delta, B\rangle \in \Lambda$, where $\Delta=\left\{A_{1}, \ldots, A_{n}\right\}$, the consecution rule

$$
\begin{array}{ccc}
0 \triangleright A_{1} & \ldots & 0 \triangleright A_{n} \\
\hline \multicolumn{3}{c}{0 \triangleright B}
\end{array}
$$

is added to B's set of consecution rules.

Sometimes theory-dependent type rules are also added to a consecution calculus. As an example, consider Priest (2015) where in the context of naïve truth theory formulated over the consecution calculus as here presented for $\mathbf{B}$, Priest suggested that a "naïve" validity predicate should be governed by the consecution rules

$$
\text { (V1) } \overline{V(\langle A\rangle,\langle B\rangle) \triangleright A \rightarrow B} \quad \text { (V2) } \frac{A \triangleright B}{0 \triangleright V(\langle A\rangle,\langle B\rangle)}
$$

Priest's naïve validity theory, then, can be represented in Hilbert-style as the pairtheory

$$
\Psi={ }_{d f}\{\langle\varnothing, V(\langle A\rangle,\langle B\rangle) \rightarrow(A \rightarrow B)\rangle,\langle\{A \rightarrow B\}, V(\langle A\rangle,\langle B\rangle)\rangle\},
$$

that is as the axiom $V(\langle A\rangle,\langle B\rangle) \rightarrow(A \rightarrow B)$ together with the theory-dependent rule $\{A \rightarrow B\} \Vdash V(\langle A\rangle,\langle B\rangle)$.

\section{Summary}

This paper has dug into Restall's consecution calculus. It was shown that his weak soundness and completeness result extends to a strong one, and that this even holds in the presence of the meta-rule of reasoning by cases, as well as when axiomatic theories come equipped with so-called theory-dependent rules.

\section{REFERENCES}

Avron, A. (1988). The semantics and proof theory of linear logic. Theoretical Computer Science 57(2), 161-184. doi: 10.1016/0304-3975(88)90037-0.

Bimbó, K. (2007). Relevance logics. In D. Jacquette (Ed.), Philosophy of Logic, Handbook of the Philosophy of Science, pp. 723-789. Amsterdam: NorthHolland. doi: 10.1016/B978-044451541-4/50022-1.

Brady, R. T. (1984). Natural deduction systems for some quantified relevant logics. Logique et Analyse 27(108), 355-377. https://www.jstor.org/stable/ 44084099.

Brady, R. T. (1993). Rules in relevant logic-II: Formula representation. Studia Logica 52(4), 565-585. doi: 10.1007/BF01053260.

Brady, R. T. (1994). Rules in relevant logic-I: Semantic classification. Journal of Philosophical Logic 23(2), 111-137. doi: 10.1007/BF01050340.

Dunn, J. M. (1979). Relevant Robinson's arithmetic. Studia Logica 38(4), 407418. doi: 10.1007/BF00370478.

Australasian Journal of Logic (18:2) 2021, Article no. 2 
Dunn, J. M. and G. Restall (2002). Relevance logic. In D. M. Gabbay and F. Guenthner (Eds.), Handbook of Philosophical Logic, Volume 6, pp. 1-128. Dordrecht: Springer Netherlands. doi: 10.1007/978-94-017-0460-1_1.

Friedman, H. and R. K. Meyer (1992). Whither relevant arithmetic? Journal of Symbolic Logic 57(3), 824-831. doi: 10.2307/2275433.

Lambek, J. (1958). The mathematics of sentence structure. The American Mathematical Monthly 65(3), 154-170. doi: 10.2307/2310058.

Mares, E. D. and R. K. Meyer (2001). Relevant logics. In The Blackwell Guide to Philosophical Logic, pp. 280-308. Malden: Blackwell Publishers Ltd. doi: 10.1002/9781405164801.ch13.

Meyer, K. R. (1976). Relevant arithmetic. Bulletin of the Section of Logic 5(4), 133-137.

Meyer, R. K. (2004). Ternary relations and relevant semantics. Annals of Pure and Applied Logic 127(1), 195 - 217. doi: 10.1016/j.apal.2003.11.015.

Meyer, R. K. and C. Mortensen (1984). Inconsistent models for relevant arithmetics. Journal of Symbolic Logic 49(3), 917-929. doi: 10.2307/2274145.

Meyer, R. K. and G. Restall (1996). Linear arithmetic desecsed. Logique $\mathcal{E}$ Analyse 39(155/156), 379-387. https://www . jstor . org/stable/44084577.

Meyer, R. K. and G. Restall (1999). "Strenge" arithmetics. Logique E Analyse 42(167/168), 205-220. https: //www. jstor .org/stable/44084657.

Øgaard, T. F. (2017). Skolem functions in non-classical logics. Australasian Journal of Logic 14(1), 181-225. doi: 10.26686/ajl.v14i1.4031.

Priest, G. (2015). Fusion and confusion. Topoi 34(1), 55-61. doi: 10.1007/s11245013-9175-X.

Priest, G. and R. Sylvan (1992). Simplified semantics for basic relevant logic. Journal of Philosophical Logic 21(2), 217-232. doi: 10.1007/BF00248640.

Read, S. (1988). Relevant Logic: A Philosophical Examination of Inference. Oxford: Basil Blackwell.

Restall, G. (1992). Arithmetic and truth in Łukasiewicz's infinitely valued logic. Logique et Analyse 139-140, 303-312. https://www.jstor.org/stable/ 44085060.

Restall, G. (1994). On Logics Without Contraction. Ph. D. thesis, The University of Queensland. https://consequently.org/writing/onlogics/.

Restall, G. (2000). An Introduction to Substructural Logics. London: Routledge. doi: $10.4324 / 9780203016244$.

Restall, G. (2006). Relevant and substructural logics. In D. M. Gabbay and J. Woods (Eds.), Logic and the Modalities in the Twentieth Century, Volume 7 of Handbook of the History of Logic, pp. 289-398. North-Holland. doi: 10.1016/S1874-5857(06)80030-0.

Restall, G. (2010). Models for substructural arithmetics. Australasian Journal of Logic 8, 82-99. doi: 10.26686/ajl.v8i0.1814.

Slaney, J. (1990). A general logic. Australasian Journal of Philosophy 68(1), 74-88. doi: 10.1080/00048409012340183. 
Slaney, J. K. (1995). MaGIC, Matrix Generator for Implication Connectives: Release 2.1 notes and guide. Technical Report TR-ARP-11/95, Automated Reasoning Project, Australian National University. http: //ftp. rsise . anu . edu . au/techreports/1995/TR-ARP-11-95.dvi.gz.

Department of Philosophy, University of Bergen

Email address: Tore.0gaard@uib.no 